Proceedings of the Edinburgh Mathematical Society (2002) 45, 579-587 (C)

DOI:10.1017/S001309150100075X Printed in the United Kingdom

\title{
ON ISOMETRIC MINIMAL IMMERSIONS FROM WARPED PRODUCTS INTO REAL SPACE FORMS
}

\author{
BANG-YEN CHEN \\ Department of Mathematics, Michigan State University, East Lansing, \\ MI 48824-1027, USA (bychen@math.msu.edu)
}

(Received 17 August 2001)

\begin{abstract}
We establish a general sharp inequality for warped products in real space form. As applications, we show that if the warping function $f$ of a warped product $N_{1} \times{ }_{f} N_{2}$ is a harmonic function, then

(1) every isometric minimal immersion of $N_{1} \times{ }_{f} N_{2}$ into a Euclidean space is locally a warped-product immersion, and

(2) there are no isometric minimal immersions from $N_{1} \times_{f} N_{2}$ into hyperbolic spaces.

Moreover, we prove that if either $N_{1}$ is compact or the warping function $f$ is an eigenfunction of the Laplacian with positive eigenvalue, then $N_{1} \times_{f} N_{2}$ admits no isometric minimal immersion into a Euclidean space or a hyperbolic space for any codimension. We also provide examples to show that our results are sharp.
\end{abstract}

Keywords: warped products; warped-product immersion; inequality; minimal immersions; real space forms; eigenfunction of the Laplacian

AMS 2000 Mathematics subject classification: Primary 53C40; 53C42; 53B25

\section{Introduction}

Let $B$ and $F$ be two Riemannian manifolds of positive dimensions equipped with Riemannian metrics $g_{B}$ and $g_{F}$, respectively, and let $f$ be a positive function on $B$. Consider the product manifold $B \times F$ with its projection $\pi: B \times F \rightarrow B$ and $\eta: B \times F \rightarrow F$. The warped product $M=B \times{ }_{f} F$ is the manifold $B \times F$ equipped with the Riemannian structure such that

$$
\|X\|^{2}=\left\|\pi_{*}(X)\right\|^{2}+f^{2}(\pi(x))\left\|\eta_{*}(X)\right\|^{2}
$$

for any tangent vector $X \in T_{x} M$. Thus, we have $g=g_{B}+f^{2} g_{F}$. The function $f$ is called the warping function of the warped product. It is well known that the notion of warped products plays some important roles in differential geometry as well as in physics. For instance, the best relativistic model of the Schwarzschild space-time that describes the outer space around a massive star or a black hole is given as a warped product (cf. [9, pp. 364-367]). (For a recent survey on warped products as Riemannian submanifolds, see [4].) 
For a warped product $N_{1} \times_{f} N_{2}$, we denote by $\mathcal{D}_{1}$ and $\mathcal{D}_{2}$ the distributions given by the vectors tangent to leaves and fibres, respectively. Thus, $\mathcal{D}_{1}$ is obtained from tangent vectors of $N_{1}$ via the horizontal lift and $\mathcal{D}_{2}$ is obtained by tangent vectors of $N_{2}$ via the vertical lift. Let $\phi: N_{1} \times_{f} N_{2} \rightarrow R^{m}(c)$ be an isometric immersion of a warped product $N_{1} \times_{f} N_{2}$ into a Riemannian manifold with constant sectional curvature $c$. Denote by $h$ the second fundamental form of $\phi$. The immersion $\phi$ is called mixed totally geodesic if $h(X, Z)=0$ for any $X$ in $\mathcal{D}_{1}$ and $Z$ in $\mathcal{D}_{2}$.

One of the most fundamental problems in the theory of submanifolds is the immersibility (or non-immersibility) of a Riemannian manifold in a Euclidean $m$-space $E^{m}$ (or, more generally, in a space form $R^{m}(c)$ of constant curvature $c$ ). According to a wellknown theorem of Nash, every Riemannian manifold can be isometrically immersed in some Euclidean spaces with sufficiently high codimension. The Nash Theorem was aimed for in the hope that if Riemannian manifolds could always be regarded as Riemannian submanifolds, this would then yield the opportunity of using extrinsic help.

Based on Nash's Theorem, one of my research programs is 'to search for control of extrinsic quantities in relation to intrinsic quantities of Riemannian manifolds via Nash's Theorem and to search for their applications'.

Since Nash's Theorem implies that every warped product $N_{1} \times_{f} N_{2}$ can always be regarded as a Riemannian submanifold in some Euclidean space, a special case of the research program is thus to study the two following fundamental problems.

Problem 1.1.

$$
\forall N_{1} \times_{f} N_{2} \underset{\text { immersion }}{\stackrel{\text { isometric }}{\longrightarrow}} E^{m} \quad \text { or } \quad R^{m}(c) \Longrightarrow ? ? ?
$$

Problem 1.2. Let $N_{1} \times_{f} N_{2}$ be an arbitrary warped product isometrically immersed in $E^{m}$ (or in $R^{m}(c)$ ) as a Riemannian submanifold. What are the relationships between the warping function $f$ and the extrinsic structures of $N_{1} \times_{f} N_{2}$ ?

In view of Nash's Theorem, it is natural to impose a natural condition on the immersibility problem. For example, if one imposes the minimality condition on the immersions, it leads to the following problem.

Problem 1.3. Given a warped product $N_{1} \times_{f} N_{2}$, what are the necessary conditions for the warped product to admit a minimal isometric immersion in a Euclidean $m$-space $E^{m}$ (or in $\left.R^{m}(c)\right)$ ?

In this paper we prove the following results, which provide some solutions to these fundamental problems.

Theorem 1.4. Let $\phi: N_{1} \times_{f} N_{2} \rightarrow R^{m}(c)$ be an isometric immersion of a warped product into a Riemannian $m$-manifold of constant sectional curvature $c$. Then we have

$$
\frac{\Delta f}{f} \leqslant \frac{\left(n_{1}+n_{2}\right)^{2}}{4 n_{2}} H^{2}+n_{1} c
$$

where $n_{i}=\operatorname{dim} N_{i}, i=1,2, H^{2}$ is the squared mean curvature of $\phi$, and $\Delta$ is the Laplacian operator of $N_{1}$. 
The equality sign of (1.2) holds identically if and only if $\phi: N_{1} \times_{f} N_{2} \rightarrow R^{m}(c)$ is a mixed totally geodesic immersion with $\operatorname{tr} h_{1}=\operatorname{tr} h_{2}$, where $\operatorname{tr} h_{1}$ and $\operatorname{tr} h_{2}$ denote the trace of $h$ restricted to $N_{1}$ and $N_{2}$, respectively.

As applications of Theorem 1.4 we have the following theorems.

Theorem 1.5. Let $N_{1} \times_{f} N_{2}$ be a warped product whose warping function $f$ is a harmonic function. Then

(1) $N_{1} \times_{f} N_{2}$ admits no isometric minimal immersion into a hyperbolic space for any codimension; and

(2) every isometric minimal immersion from $N_{1} \times_{f} N_{2}$ into a Euclidean space is a warped-product immersion.

Theorem 1.6. If $f$ is an eigenfunction of the Laplacian on $N_{1}$ with eigenvalue $\lambda>0$, then $N_{1} \times{ }_{f} N_{2}$ does not admit an isometric minimal immersion into a Euclidean space or a hyperbolic space for any codimension.

Theorem 1.7. If $N_{1}$ is a compact Riemannian manifold, then every warped product $N_{1} \times_{f} N_{2}$ does not admit an isometric minimal immersion into a Euclidean space or a hyperbolic space for any codimension.

In the last section, we provide some examples to show that these results are best possible.

\section{Preliminaries}

Let $N$ be an $n$-dimensional submanifold of a Riemannian $m$-manifold $R^{m}(c)$ of constant sectional curvature $c$. We choose a local field of orthonormal frame

$$
e_{1}, \ldots, e_{n}, e_{n+1}, \ldots, e_{m} \text { in } R^{m}(c)
$$

such that, restricted to $N$, the vectors $e_{1}, \ldots, e_{n}$ are tangent to $N$ and $e_{n+1}, \ldots, e_{m}$ are normal to $N$.

Let $K\left(e_{i} \wedge e_{j}\right), 1 \leqslant i<j \leqslant n$, denote the sectional curvature of the plane section spanned by $e_{i}$ and $e_{j}$. Then the scalar curvature of $N$ is given by

$$
\tau=\sum_{i<j} K\left(e_{i} \wedge e_{j}\right)
$$

For a submanifold $N$ in $R^{m}(c)$ we denote by $\nabla$ and $\tilde{\nabla}$ the Levi-Civita connections of $N$ and $R^{m}(c)$, respectively. The Gauss and Weingarten formulae are given, respectively, by

$$
\begin{aligned}
\tilde{\nabla}_{X} Y & =\nabla_{X} Y+h(X, Y), \\
\tilde{\nabla}_{X} \xi & =-A_{\xi} X+D_{X} \xi
\end{aligned}
$$


for vector fields $X, Y$ tangent to $N$ and $\xi$ normal to $N$, where $h$ denotes the second fundamental form, $D$ the normal connection, and $A$ the shape operator of the submanifold. Let $\left\{h_{i j}^{r}\right\}, i, j=1, \ldots, n, r=n+1, \ldots, m$, denote the coefficients of the second fundamental form $h$ with respect to $e_{1}, \ldots, e_{n}, e_{n+1}, \ldots, e_{m}$.

The mean curvature vector $\boldsymbol{H}$ is defined by

$$
\boldsymbol{H}=\frac{1}{n} \operatorname{tr} h=\frac{1}{n} \sum_{i=1}^{n} h\left(e_{i}, e_{i}\right)
$$

where $\left\{e_{1}, \ldots, e_{n}\right\}$ is a local orthonormal frame of the tangent bundle $T N$ of $N$. The squared mean curvature is given by $H^{2}=\langle\boldsymbol{H}, \boldsymbol{H}\rangle$, where $\langle\cdot, \cdot\rangle$ denotes the inner product. A submanifold $N$ is called minimal in $R^{m}(c)$ if the mean curvature vector of $N$ in $R^{m}(c)$ vanishes identically.

Denote by $R$ the Riemann curvature tensor of $N$. Then the equation of Gauss is given by (see, for example, [1] )

$$
\begin{aligned}
R(X, Y ; Z, W)=c\{\langle X, W\rangle\langle Y, Z\rangle-\langle X, Z\rangle\langle Y, W\rangle\} & \\
+ & \langle h(X, W), h(Y, Z)\rangle-\langle h(X, Z), h(Y, W)\rangle,
\end{aligned}
$$

for vectors $X, Y, Z, W$ tangent to $N$.

Let $M$ be a Riemannian $p$-manifold and $\left\{e_{1}, \ldots, e_{p}\right\}$ be an orthonormal frame field on $M$. For a differentiable function $\varphi$ on $M$, the Laplacian of $\varphi$ is defined by

$$
\Delta \varphi=\sum_{j=1}^{p}\left\{\left(\nabla_{e_{j}} e_{j}\right) \varphi-e_{j} e_{j} \varphi\right\} .
$$

Recall that if $M$ is compact, every eigenvalue of $\Delta$ is non-negative.

Let $\phi: N_{1} \times_{f} N_{2} \rightarrow R^{m}(c)$ denote an isometric immersion of a warped product $N_{1} \times{ }_{f} N_{2}$ into a Riemannian manifold with constant sectional curvature $c$. Denote by $\operatorname{tr} h_{1}$ and $\operatorname{tr} h_{2}$ the trace of $h$ restricted to $N_{1}$ and $N_{2}$, respectively, that is

$$
\operatorname{tr} h_{1}=\sum_{\alpha=1}^{n_{1}} h\left(e_{\alpha}, e_{\alpha}\right), \quad \operatorname{tr} h_{2}=\sum_{t=n_{1}+1}^{n_{1}+n_{2}} h\left(e_{t}, e_{t}\right)
$$

for some orthonormal frame fields $e_{1}, \ldots, e_{n_{1}}$ and $e_{n_{1}+1}, \ldots, e_{n_{1}+n_{2}}$ of $\mathcal{D}_{1}$ and $\mathcal{D}_{2}$, respectively.

If $M_{1} \times{ }_{\rho} M_{2}$ is a warped product of two Riemannian manifolds and $\phi_{i}: N_{i} \rightarrow M_{i}$, $i=1,2$, are isometric immersions from Riemannian manifolds $N_{1}, N_{2}$ into Riemannian manifolds $M_{1}, M_{2}$, respectively. Define a positive function $\sigma$ on $N_{1}$ by $\sigma=\rho \circ \phi_{1}$. Then the map

$$
\phi: N_{1} \times{ }_{\sigma} N_{2} \rightarrow M_{1} \times{ }_{\rho} M_{2}
$$

given by $\phi\left(x_{1}, x_{2}\right)=\left(\phi_{1}\left(x_{1}\right), \phi_{2}\left(x_{2}\right)\right)$ is an isometric immersion, which is called a warpedproduct immersion $[\mathbf{8}]$ (see also [5]). 


\section{Proof of Theorem 1.4}

Let $\phi: N=N_{1} \times_{f} N_{2} \rightarrow R^{m}(c)$ be an isometric immersion of a warped product $N_{1} \times{ }_{f} N_{2}$ into a Riemannian manifold of constant sectional curvature $c$. Denote by $n_{1}, n_{2}, n$ the dimensions of $N_{1}, N_{2}, N_{1} \times N_{2}$, respectively.

Since $N_{1} \times_{f} N_{2}$ is a warped product, we have

$$
\nabla_{X} Z=\nabla_{Z} X=(X \ln f) Z
$$

for unit vector fields $X, Z$ tangent to $N_{1}, N_{2}$, respectively. Hence, we find

$$
\begin{aligned}
K(X \wedge Z) & =\left\langle\nabla_{Z} \nabla_{X} X-\nabla_{X} \nabla_{Z} X, Z\right\rangle \\
& =(1 / f)\left\{\left(\nabla_{X} X\right) f-X^{2} f\right\} .
\end{aligned}
$$

If we chose a local orthonormal frame $e_{1}, \ldots, e_{n}$ such that $e_{1}, \ldots, e_{n_{1}}$ are tangent to $N_{1}$ and $e_{n_{1}+1}, \ldots, e_{n}$ are tangent to $N_{2}$, then we have

$$
\frac{\Delta f}{f}=\sum_{j=1}^{n_{1}} K\left(e_{j} \wedge e_{s}\right)
$$

for each $s=n_{1}+1, \ldots, n$.

From the equation of Gauss, it follows that the scalar curvature $\tau$ and the squared mean curvature $H^{2}$ of $N$ satisfy

$$
2 \tau=n^{2} H^{2}-\|h\|^{2}+n(n-1) c,
$$

where $\|h\|^{2}$ is the squared norm of the second fundamental form $h$ of $N$ in $R^{m}(c)$.

Let us put

$$
\delta=2 \tau-n(n-1) c-\frac{1}{2} n^{2} H^{2} .
$$

Then (3.4) becomes

$$
n^{2} H^{2}=2 \delta+2\|h\|^{2}
$$

If we choose an orthonormal frame $e_{n+1}, \ldots, e_{m}$ of the normal bundle so that $e_{n+1}$ is in the direction of the mean curvature vector, then (3.6) becomes

$$
\left(\sum_{i=1}^{n} h_{i i}^{n+1}\right)^{2}=2\left[\delta+\sum_{i=1}^{n}\left(h_{i i}^{n+1}\right)^{2}+\sum_{i \neq j}\left(h_{i j}^{n+1}\right)^{2}+\sum_{r=n+2}^{m} \sum_{i, j=1}^{n}\left(h_{i j}^{r}\right)^{2}\right] .
$$

Equation (3.7) is equivalent to

$$
\begin{aligned}
\left(\bar{a}_{1}+\bar{a}_{2}+\bar{a}_{3}\right)^{2}=2\left[\delta+\bar{a}_{1}^{2}+\right. & \bar{a}_{2}^{2}+\bar{a}_{3}^{2}+2 \sum_{1 \leqslant i<j \leqslant n}\left(h_{i j}^{n+1}\right)^{2}+\sum_{r=n+2}^{m} \sum_{i, j=1}^{n}\left(h_{i j}^{r}\right)^{2} \\
& \left.-2 \sum_{2 \leqslant j<k \leqslant n_{1}} h_{j j}^{n+1} h_{k k}^{n+1}-2 \sum_{n_{1}+1 \leqslant s<t \leqslant n} h_{s s}^{n+1} h_{t t}^{n+1}\right],
\end{aligned}
$$


where

$$
\bar{a}_{1}=h_{11}^{n+1}, \quad \bar{a}_{2}=h_{22}^{n+1}+\cdots+h_{n_{1} n_{1}}^{n+1}, \quad \bar{a}_{3}=h_{n_{1}+1 n_{1}+1}^{n+1}+\cdots+h_{n n}^{n+1} .
$$

Applying Lemma 3.1 of $[\mathbf{2}]$ or of $[\mathbf{3}]$ to (3.8) yields

$$
\begin{aligned}
\sum_{1 \leqslant j<k \leqslant n_{1}} h_{j j}^{n+1} h_{k k}^{n+1}+ & \sum_{n_{1}+1 \leqslant s<t \leqslant n} h_{s s}^{n+1} h_{t t}^{n+1} \\
& \geqslant \frac{1}{2} \delta+\sum_{1 \leqslant \alpha<\beta \leqslant n}\left(h_{\alpha \beta}^{n+1}\right)^{2}+\frac{1}{2} \sum_{r=n+2}^{m} \sum_{\alpha, \beta=1}^{n}\left(h_{\alpha \beta}^{r}\right)^{2},
\end{aligned}
$$

with equality holding if and only if we have

$$
h_{11}^{n+1}+\cdots+h_{n_{1} n_{1}}^{n+1}=h_{n_{1}+1 n_{1}+1}^{n+1}+\cdots+h_{n n}^{n+1} .
$$

From the equation of Gauss and (3.3), we have

$$
\begin{aligned}
\frac{n_{2} \Delta f}{f}=\tau & -\sum_{1 \leqslant j<k \leqslant n_{1}} K\left(e_{j} \wedge e_{k}\right)-\sum_{n_{1}+1 \leqslant s<t \leqslant n} K\left(e_{s} \wedge e_{t}\right) \\
= & -\frac{1}{2}\left(n_{1}\left(n_{1}-1\right)\right) c-\sum_{r=n+1}^{m} \sum_{1 \leqslant j<k \leqslant n_{1}}\left(h_{j j}^{r} h_{k k}^{r}-\left(h_{j k}^{r}\right)^{2}\right) \\
& -\frac{1}{2}\left(n_{2}\left(n_{2}-1\right)\right) c-\sum_{r=n+1}^{m} \sum_{n_{1}+1 \leqslant s<t<n}\left(h_{s s}^{r} h_{t t}^{r}-\left(h_{s t}^{r}\right)^{2}\right) .
\end{aligned}
$$

Therefore, by (3.5), (3.10) and (3.12), we obtain

$$
\begin{aligned}
& \frac{n_{2} \Delta f}{f} \leqslant \tau-\frac{1}{2}(n(n-1)) c+n_{1} n_{2} c-\frac{1}{2} \delta-\sum_{\substack{1 \leqslant j \leqslant n_{1} ; \\
n_{1}+1 \leqslant t \leqslant n}}\left(h_{j t}^{n+1}\right)^{2} \\
& -\frac{1}{2} \sum_{r=n+2}^{m} \sum_{\alpha, \beta=1}^{n}\left(h_{\alpha \beta}^{r}\right)^{2}+\sum_{r=n+2}^{m} \sum_{1 \leqslant j<k \leqslant n_{1}}\left(\left(h_{j k}^{r}\right)^{2}-h_{j j}^{r} h_{k k}^{r}\right) \\
& \quad+\sum_{r=n+2}^{m} \sum_{n_{1}+1 \leqslant s<t<n}\left(\left(h_{s t}^{r}\right)^{2}-h_{s s}^{r} h_{t t}^{r}\right) \\
& =\tau-\frac{1}{2}(n(n-1)) c+n_{1} n_{2} c-\frac{1}{2} \delta-\sum_{r=n+1}^{m} \sum_{1 \leqslant j \leqslant n_{1}} \sum_{n_{1}+1 \leqslant t \leqslant n}\left(h_{j t}^{r}\right)^{2} \\
& \quad-\frac{1}{2} \sum_{r=n+2}^{m}\left(\sum_{1 \leqslant j \leqslant n_{1}} h_{j j}^{r}\right)^{2}-\frac{1}{2} \sum_{r=n+2}^{m}\left(\sum_{n_{1}+1 \leqslant t \leqslant n} h_{t t}^{r}\right)^{2} \\
& \leqslant \tau-\frac{1}{2}(n(n-1)) c+n_{1} n_{2} c-\frac{1}{2} \delta \\
& =\frac{1}{4} n^{2} H^{2}+n_{1} n_{2} c,
\end{aligned}
$$


which proves inequality (1.2). From (3.11) and (3.13) we know that the equality sign of (1.2) holds if and only if

$$
h_{j t}^{r}=0 \quad \text { for } n+1 \leqslant r \leqslant m,
$$

and

$$
h_{11}^{r}+\cdots+h_{n_{1} n_{1}}^{r}=h_{n_{1}+1 n_{1}+1}^{r}+\cdots+h_{n n}^{r}=0
$$

for $1 \leqslant j \leqslant n_{1}, n_{1}+1 \leqslant t \leqslant n, n+2 \leqslant r \leqslant m$.

Condition (3.14) implies that the second fundamental form of $N_{1} \times_{f} N_{2}$ in $R^{m}(c)$ satisfies $h\left(\mathcal{D}_{1}, \mathcal{D}_{2}\right)=\{0\}$. Thus, the immersion $\phi$ is mixed totally geodesic. Hence, by applying a result of Nölker [8], we know that, locally, there exists a warped-product representation $M_{1} \times{ }_{\rho} M_{2}$ of $R^{m}(c)$ such that $\phi: N_{1} \times{ }_{f} N_{2} \rightarrow M_{1} \times{ }_{\rho} M_{2}=R^{m}(c)$ is a warpedproduct immersion of $\phi_{1}: N_{1} \rightarrow M_{1}$ and $\phi_{2}: N_{2} \rightarrow M_{2}$; so that we have $\phi\left(x_{1}, x_{2}\right)=$ $\left(\phi_{1}\left(x_{1}\right), \phi\left(x_{2}\right)\right)$, for $x_{1} \in N_{1}, x_{2} \in N_{2}$. Moreover, from (3.11) and (3.15), we obtain

$$
\sum_{j=1}^{n_{1}} h\left(e_{j}, e_{j}\right)=\sum_{s=n_{1}+1}^{n_{1}+n_{2}} h\left(e_{s}, e_{s}\right) .
$$

Hence, we have $\operatorname{tr} h_{1}=\operatorname{tr} h_{2}$.

Conversely, suppose that $\phi: N_{1} \times_{f} N_{2} \rightarrow M_{1} \times_{\rho} M_{2}=R^{m}(c)$ is a mixed totally geodesic immersion with $\operatorname{tr} h_{1}=\operatorname{tr} h_{2}$. Then all the inequalities in (3.10), (3.13) become equalities. Hence, by (3.13) we obtain the equality sign of (1.2).

\section{Proofs of Theorems 1.5-1.7}

Assume that $\phi: N_{1} \times_{f} N_{2} \rightarrow R^{m}(c)$ is an isometric minimal immersion of a warped product $N_{1} \times_{f} N_{2}$ into a complete simply connected Riemannian manifold $R^{m}(c)$ of constant sectional curvature $c$.

If $f$ is a harmonic function on $N_{1}$, then inequality (1.2) of Theorem 1.4 implies $c \geqslant 0$. In particular, this shows that the warped product $N_{1} \times_{f} N_{2}$ does not admit any isometric minimal immersion into a hyperbolic space.

When $c=0$. The minimality of $N_{1} \times_{f} N_{2}$ and the harmonicity of $f$ imply that the equality sign of (1.2) holds identically. Thus, the immersion is mixed totally geodesic according to Theorem 1.4. Hence, by applying a result of [8], we know that $\phi$ is locally a warped-product immersion. This proves Theorem 1.5.

When $f$ is an eigenfunction of the Laplacian on $N_{1}$ with eigenvalue $\lambda>0$. Then inequality (1.2) implies that $n_{1} c \geqslant \lambda>0$. Hence, the ambient space $R^{m}(c)$ cannot be a Euclidean space or a hyperbolic space. Therefore, we have Theorem 1.6.

Now, we assume that $N_{1}$ is compact and $\phi: N_{1} \times{ }_{f} N_{2} \rightarrow R^{m}(c)$ is an isometric minimal immersion with $c \leqslant 0$. Then, inequality $(1.2)$ implies that $(\Delta f) / f \leqslant 0$. Since the warping function is a positive function, we have $\Delta f \leqslant 0$. Hence, by applying Hopf's Lemma and the compactness of $N_{1}$, we know that $f$ is a positive constant. Therefore, the warped product $N_{1} \times_{f} N_{2}$ is a Riemannian product of the Riemannian manifold $\left(N_{1}, g_{1}\right)$ and the 
Riemannian manifold $\left(N_{2}, f^{2} g_{2}\right)$, equipped with the metric $f^{2} g_{2}$ which is homothetic to the original metric $g_{2}$ on $N_{2}$. Because $f$ is constant and $\phi: N_{1} \times_{f} N_{2} \rightarrow R^{m}(c)$ is an isometric minimal immersion, inequality (1.2) implies $c=0$.

By using the constancy of $f$, minimality of the warped product, and $c=0$, we obtain the equality sign of (1.2). Hence, the immersion is mixed totally geodesic. Therefore, by applying a result of Moore [7], we know that $\phi$ is a product immersion, say

$$
\phi=\left(\phi_{1}, \phi_{2}\right):\left(N_{1}, g_{1}\right) \times\left(N_{2}, f^{2} g_{2}\right) \rightarrow E^{m_{1}} \times E^{m_{2}}=E^{m} .
$$

Since $\phi$ is minimal in $E^{m}$, the first factor $\phi_{1}: N_{1} \rightarrow E^{m_{1}}$ is also an isometric minimal immersion, which is impossible due to compactness of $N_{1}$. Hence, when $N_{1}$ is compact, $N_{1} \times_{f} N_{2}$ does not admit any isometric minimal immersions into Euclidean space and hyperbolic space regardless of codimension.

\section{Some remarks}

In view of Theorems 1.4-1.7, we provide the following remarks.

Remark 5.1. There exist many minimal submanifolds in Euclidean space which are warped products with harmonic warping function. For example, if $N_{2}$ is a minimal submanifold of the unit $(m-1)$-sphere $S^{m-1} \subset E^{m}$, the minimal cone $C\left(N_{2}\right)$ over $N_{2}$ with vertex at the origin of $E^{m}$ is the warped product $R_{+} \times{ }_{s} N_{2}$ whose warping function $f=s$ is a harmonic function. Here $s$ is the coordinate function of the positive real line $R_{+}$.

Remark 5.2. In view of Theorem 1.6, it is interesting to point out that there exist isometric minimal immersions from warped products $N_{1} \times_{f} N_{2}$ into a hyperbolic space such that the warping function $f$ is an eigenfunction with negative eigenvalue. For example, $R \times{ }_{e^{x}} E^{n-1}$ admits an isometric minimal immersion into the hyperbolic space $H^{n+1}(-1)$ of constant sectional curvature -1 .

Remark 5.3. In view of Theorem 1.7, it is interesting to point out that there do exist many isometric minimal immersions from $N_{1} \times{ }_{f} N_{2}$ into Euclidean space with compact $N_{2}$. For example, a hypercaternoid in $E^{n+1}$ is a minimal hypersurface which is isometric to a warped product $R \times_{f} S^{n-1}$. Also, for any compact minimal submanifold $N_{2}$ of $S^{m-1} \subset E^{m}$, the minimal cone $C\left(N_{2}\right)$ is a warped product $R_{+} \times_{s} N_{2}$, which is also such an example.

Remark 5.4. In contrast to Euclidean and hyperbolic spaces, the standard $m$-sphere $S^{m}$ admits warped-product minimal submanifolds $N_{1} \times_{f} N_{2}$ such that $N_{1}, N_{2}$ are both compact. The simplest of such examples are minimal Clifford tori $M_{k, n-k}, k=2, \ldots, n-$ 1 , in $S^{n+1}$ defined by

$$
M_{k, n-k}=S^{k}\left(\sqrt{\frac{k}{n}}\right) \times S^{n-k}\left(\sqrt{\frac{(n-k)}{n}}\right) .
$$

Remark 5.5. Ejiri constructed in $[\mathbf{6}]$ many examples of warped-product minimal immersions into complete simply connected Riemannian manifolds of constant sectional curvature. 
The above remarks show that Theorems 1.4-1.7 are best possible.

Remark 5.6. Problem 1.2 has also been studied in [10] from a different aspect for the class of warped products.

Remark 5.7. Inequality (1.2) also holds for warped products isometrically immersed in complex hyperbolic $m$-space $\mathrm{CH}^{m}(4 c)$ of constant holomorphic sectional curvature $4 c<0$ as well as for warped products isometrically immersed in complex projective $m$-space $\mathrm{CP}^{m}(4 c)$ as totally real submanifolds.

Remark 5.8. The same proof as for Theorem 1.6 shows that for any positive function $f$ on $N_{1}$ with $(\Delta f) / f$ being positive at some points, the warped product $N_{1} \times{ }_{f} N_{2}$ does not admit an isometric minimal immersion into Euclidean space or hyperbolic space for any codimension.

\section{References}

1. B. Y. Chen, Geometry of submanifolds (Marcel Dekker, New York, 1973).

2. B. Y. CHEN, Some pinching and classification theorems for minimal submanifolds, Arch. Math. 60 (1993), 568-578.

3. B. Y. CHEN, Some new obstructions to minimal and Lagrangian isometric immersions, Jpn. J. Math. 26 (2000), 105-127.

4. B. Y. Chen, Geometry of warped products as Riemannian submanifolds and related problems, Soochow J. Math. 28 (2002), 125-156.

5. F. Dillen And S. Nölker, Semi-parallelity, multi-rotation surfaces and the helixproperty, J. Reine Angew. Math. 435 (1993), 33-63.

6. N. EJIRI, A generalization of minimal cones, Trans. Am. Math. Soc. 276 (1983), 347-360.

7. J. D. Moore, Isometric immersions of Riemannian products, J. Diff. Geom. 5 (1971), $159-168$.

8. S. NÖLKER, Isometric immersions of warped products, Diff. Geom. Applic. 6 (1996), 1-30.

9. B. O'NeILl, Semi-Riemannian geometry with applications to relativity (Academic, 1983).

10. B. SucEAvĂ, The Chen invariants of warped products of hyperbolic planes and their applications to immersibility problems, Tsukuba J. Math. 25 (2001), 311-320. 